\title{
Basketball scoring system simulation
}

\author{
Dong Gang
}

Chongqing college of engineering

Department of electronic and information engineering, Chongqing 400056 ,China

240463401@qq.com

\begin{abstract}
.
In order to achieve the display effect of basketball scoring system, this paper proposes a Proteus simulation test system design scheme based on single chip microcomputer, and completes the hardware and software design of the system. The hardware part mainly USES the Proteus simulation, virtual instrument software part adopts singlechip c language programming, able to complete visualization simulation of the whole system. Practical application shows that the simulation circuit has the characteristics of simple operation, accurate test, meet the requirements of the system design.
\end{abstract}

Keywords: Basketball scoring system; The simulation; Proteus;Single chip microcomputer;

\section{Introduction}

With the wide application of single-chip computer in various fields, many do with single-chip computer control of the game time scoring system should also, such as using single-chip microcomputer control LCD liquid crystal display timing 14-year-old, using single-chip microcomputer control LED seven-segment display timing score indicator, etc.

This system is designed with 51 series microcontroller programming control LED seven-segment digital tube display timing game scoring system. The 
system has a schedule timing Settings, schedule time to pause, timely refresh both parties results have been staging and after the game, and other functions. It has a low price, stable performance, easy to operate and easy to carry, etc. Suitable for all kinds of schools or small groups as schedule timing scoring.

Through basketball timing score indicator design, we can understand and familiar with the single chip microcomputer development design process, and to deepen the understanding and application of single-chip microcomputer and single-chip microcomputer and peripheral interface of some methods and skills, which is mainly manifested in the following aspects:

(1) the basketball game time scoring system contains a minimum of 8051 series single chip microcomputer application system, at the same time, on the basis of the extended some peripheral interface usability is strong.

(2) to the LED display of the structure, working principle and the display interface instance with a specific connection and programming method.

(3) how to use serial port to extend the display interface, etc.

\section{System design and analysis}

Basketball scoring system of the specific design requirements are as follows:

(1) to record the course of the game, and can set the game before the start of the game, the game process can pause the game time.

(2) can refresh a, b two teams score in the entire schedule of party a and party $\mathrm{b}$ both teams score points and subtract points.

(3) the midfielder exchange venue, can exchange the position of the a, b two teams score.

(4) at the end of the game can send out alarm prompt.

(5) at every time of clearing the ball after 24 seconds to manual initialization, attack more than 24 seconds timer stop know press continue to start the time. 


\section{The overall design}

The system structure diagram as shown in figure 1, the controller with 51 series microcontroller, through the key module as input ports, adjust the time or score of digital tube, digital tube display and control the existing time or score.

The system hardware consists of the following three parts:

(1) processor: 51 series microcontroller

(2) according to some

(3) the key switch

Processor: this system adopts the 51 series single chip microcomputer as the core component of this system, compatible with MCS - 51 instruction system, 32 bidirectional I/O port, two 16 bit programmable timer/counter, a serial port interrupt, two external interrupt source, low power consumption and power down mode, free $4 \mathrm{k}$ can be repeatedly wipe (> 1000) Flash ROM, the static operation 0 to $24 \mathrm{MHZ}, 128$ x8bit internal RAM, a total of six interrupt source, enough to meet the requirements of the design.

Display module: in the system, a total of 12 access 7, a total of Yin LED display, six of them used for recording points a, b two teams, each team 3 LED display scores range can reach 0-999, enough to meet the needs of the schedule. The other six LED display is used for recording schedule time. Minutes and seconds, attack time, each with two use LED display. Which show minutes shows the time of the two Numbers and attack four leds can be adjusted by button to set. When the time set, press the start timing button at the beginning of the start timing game. Minutes and attacking time can set the range of 0-99. According to the design, timing range of 0-99 minutes, attack time 99 seconds maximum also fully meet the needs of the schedule.

Key modules: the share 10 each button design. Four of them each to adjust two teams score, party a and party b each team with two buttons, respectively, to 
add 1 score points and minus 1; 2 minutes of each button to set the game time of the two key control minutes 10 and bits respectively; The same two buttons to set the attack time ten and bits; The remaining two keys used to control the game time of start and stop, another attack is used to control time, when the pause button to press the game, the game time has the original state to another state, offensive adjustment buttons are in the exchange of the ball, manual time initial value to give offense.

When the end of a game, pause/start button can also complete the exchange of two teams score function.

\section{Proteus simulation circuit diagram}

Diversified simulation circuit, the system adopts dynamic digital tube display mode, through the node connection wiring, is the overall look beautiful and easy, the effect is more obvious, and give the feeling on the vision more realistic, without the actual device. On the effect of algorithm to achieve consistent with the actual, the test on time.

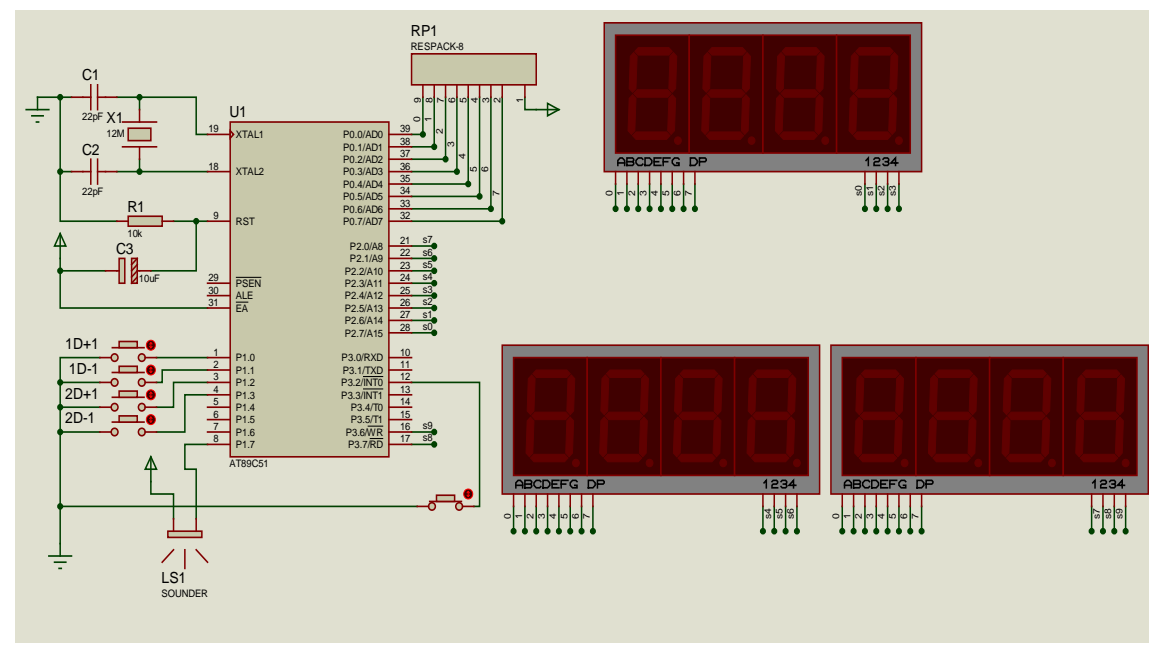

Figure 2 system simulation diagram

Direct load simulation system algorithm design code generated HEX file, 
with the actual running effect is consistent, without redundant operation, convenient beginners applications.

The test system software design

The test system software adopts C programming in a high-level language, programming platform is keil, keil platform is the common software development platform for free. The platform not only provides support for $\mathrm{C}$ language and assembly language, but also has all kinds of test, the ability of communication, control, and numerical analysis, have control, powerful function library function rich, strong real-time performance, easy in programming. In order to update, maintain and expand in the future, in the design process by adopting the idea of modularization, the whole system by key points module, timing module, display module, timer interrupt module and initialization module, etc.

System implementation process is as follows:

In the one, in the isoelectric point, the system initialization. Waiting for the time setting.

Second,when the time setting is finished, press the start button, the system displays the score and the game time.

Three, attack time by setting down to zero. The whole system suspend time, until the start button to press. Attack time starts to assignment, continue to timing.

Four, when press the pause button, the initialization attack time, stop time, waiting for the clock button to press.

Five, at the end of the countdown, issue a warning for 10 seconds.

Six, in the process of the whole time, can be modified to party a and party b both teams score. 


\section{The experimental application}

Debugging process need to pay attention to the following:

(1) the attachment is correct, before electricity should first check the circuit connection is correct, including the fault lines, lines to less or more. Lookup method: according to the circuit diagram to check the path of the installation, the characteristics of this method is that according to the circuit diagram to determine, according to certain order one by one check the installed circuit, thus, can be less easy to detect fault line and the line, there is a kind of method is carried out in accordance with the actual circuit to control principle of the circuit check line, this is a kind of method to check the line for the center with components. Each component pin connection to a check, check whether there is on each of the connection in the circuit diagram, this method can not only detect fault line and the line less, also easy to investigate more line.

(2) components when installation, check the yuan, and apparatus for short circuit between pins; Joint have poor contact; Whether diode and capacitor polarity connection; Power supply, signal source connection is correct.

(3) the power end of whether there is a short circuit, before electricity, disconnect the power cord, using a multimeter to check the power supply to end if there is a short circuit

Records on the results of simulation debugging is as follows: after the electricity, the initial display "100-100". According to the keyboard keys, distribution to $\mathrm{a}, \mathrm{b}+1$ points, 2 points and three points, the display tracking shows the total score, then detail simulation game, given a, b plus or minus points, achieves the desired effect. In both party a and b have a total score of the points a, b, and when reduced to 0 points will no longer for operation, consistent with the program design. In both party a and $b$ under the condition of a certain score, press the reset button, display the results reset. Repeat the above operation, 
the results meet the design requirements.

\section{Conclusion}

The test system adopts the common device for hardware platform, software design of the idea of modular design, improve the reliability and maintainability of the system. The test system has been used in the basketball match, the actual application shows that this test system has the test accurate, stable and reliable, friendly man-machine interface and other characteristics, has reached the design requirements.

\section{Acknowledgement}

In this paper, the research was sponsored by Chongqing city board of education science and technology research projects, based on adaptive predictive fuzzy control greenhouse system modeling research, (Project No. KJ133501) and Chongqing college of engineering for 13 years campus research projects(Project No. 2013xzky05).

\section{References}

[1] Application of Proteus Virtual System Modelling (VSM) in Teaching of Microcontroller[A]. 2010 International Conference on E-Health Networking, Digital Ecosystems and Technologies Proceedings(Volume 2)[C]. 2010

[2] Design of MCU experimental Simulation Platform Based Proteus Software[A]. Advances in Biomedical Engineering - - 2012 International Conference on Mechanical Engineering and Automation(ICMEA2012)[C]. 2012

[3] Proceedings of 2010 Third International Conference on Education Technology and Training(Volume 8)[C]. 2010

[4] Proceedings of 2010 Third International Conference on Education Technology and Training(Volume 6)[C]. 2010

[5] Reform on Experiment Teaching of Single-chip Microcomputer and Cultivation of Students' Practical Ability[A]. 2010 International Conference on E-Health Networking, Digital Ecosystems and Technologies 
Proceedings(Volume 2)[C]. 2010

[6] Advances in Artificial Intelligence (Volume 4) - - Proceedings of 2011 International Conference on Management Science and Engineering (MSE 2011)[C]. 2011

[7] Proceedings of 2010 International Conference on Broadcast Technology and Multimedia Communication(Volume 4)[C]. 2010

[8] Proceedings of 2010 Third International Conference on Education Technology and Training(Volume 8)[C]. 2010 